Wright State University

CORE Scholar

7-1-1991

\title{
Infrared Quenching and Thermal Recovery of Thermally Stimulated Current Spectra in GaAs
}

\author{
Z-Q. Fang \\ David C. Look \\ Wright State University - Main Campus, david.look@wright.edu
}

Follow this and additional works at: https://corescholar.libraries.wright.edu/physics

Part of the Physics Commons

\footnotetext{
Repository Citation

Fang, Z., \& Look, D. C. (1991). Infrared Quenching and Thermal Recovery of Thermally Stimulated Current Spectra in GaAs. Applied Physics Letters, 59 (1), 48-50.

https://corescholar.libraries.wright.edu/physics/39

This Article is brought to you for free and open access by the Physics at CORE Scholar. It has been accepted for inclusion in Physics Faculty Publications by an authorized administrator of CORE Scholar. For more information, please contact library-corescholar@wright.edu.
} 


\title{
Infrared quenching and thermal recovery of thermally stimulated current spectra in GaAs
}

\author{
Z-Q. Fang and D. C. Look \\ Physics Department, Wright State University, Dayton, Ohio 45435
}

(Received 18 February 1991; accepted for publication 1 April 1991)

\begin{abstract}
Thermally stimulated current (TSC) spectra stimulated by infrared ( $h v \leqslant 1.12 \mathrm{eV}$ ) light at 90
$\mathbf{K}$ have been used to study the photoquenching and thermal recovery of several dominant

TSC peaks in Ga-rich, semi-insulating GaAs. The filling-pulse-length dependence of the quenching for these traps, and the temperature dependence of their recovery are clearly identified with the ground-to-metastable state transition of EL2. The data are consistent with the traps having a direct association with EL2 or EL2* rather than an indirect association which could result from a change in the dominant-free carriers as EL2 transforms to EL2*. If so, they likely are various point defects or impurities complexed with the arsenic antisite.
\end{abstract}

The midgap donor level known as EL2 is responsible for the semi-insulating (SI) nature of undoped GaAs materials. Perhaps the most prominent characteristics of EL2 are its photoquenching and thermal or optical recovery behavior which have been observed by photocapacitance, ${ }^{1}$ photoluminescence, ${ }^{2}$ photoconductivity, ${ }^{3}$ infrared absorption, ${ }^{4}$ and electron paramagnetic resonance (EPR). ${ }^{5}$ The photoquenching behavior is ascribed to the metastability of EL2, i.e., the transformation of EL2 from the normal state to a metastable state (EL2*) by white or 1.1 $\mathrm{eV}$ light illumination at low temperatures $(T \leqslant 100 \mathrm{~K})$, and the thermal or optical recovery is associated with the reverse transformation, EL2* $\rightarrow$ EL2. There is a general consensus that EL2 contains the arsenic antisite $\left(A s_{G a}\right)$, but there is uncertainty as to whether it is an isolated $\mathrm{As}_{\mathrm{Ga}}$ or a complex defect, such as $\mathrm{As}_{\mathrm{Ga}}-V_{\mathrm{As}}, \mathrm{As}_{\mathrm{Ga}}-\mathrm{As}_{i}$, or $\mathrm{As}_{\mathrm{Ga}}-V_{\mathrm{Ga}}-V_{\mathrm{As}}$; the same uncertainties exist, of course, for the atomic configuration of EL2*, which has not even been detected by electrical or optical means. ${ }^{6}$ The situation is further aggravated by the paucity of data on other point defects during the quenching and recovery processes. In one of the few such studies, EPR data have revealed several acceptors like FR 3 and BE1 in as-grown and annealed SI-GaAs respectively, after the photoquenching of EL2 at $4 \mathrm{~K}$ or below. 7,8

In this letter we report a reversible change in the spectral structure of thermally stimulated current (TSC) spectra during the processes of infrared photoquenching of EL2 and thermal recovery of EL2*. By checking the quenching and enhancement of relevant traps, which are believed to be related to individual point defects or their complexes, and comparing the photocurrent quenching with TSC quenching, we have found that EL2 controls the behavior of these traps. They likely are directly associated with EL2 or possibly other $\mathrm{As}_{\mathrm{Ga}}$ complexes.

The undoped SI-GaAs sample used in this study was cut from a GaAs ingot grown by a high-pressure liquid encapsulated Czochralski (LEC) technique using a Garich melt, and annealed at $950^{\circ} \mathrm{C}$. The EL2 concentration measured by $1.1 \mu \mathrm{m}$ absorption was $7.4 \times 10^{15} \mathrm{~cm}^{-3}$, the carbon concentration determined by $582 \mathrm{~cm}^{-1}$ local vibrational mode absorption was $1 \times 10^{15} \mathrm{~cm}^{-3}$, and the dark current in the temperature range from 250 to $360 \mathrm{~K}$ was shown to be contralled by EL2, as deduced from the 0.74 $\mathrm{eV}$ activation energy. ${ }^{9}$ A rectangular Hall bar sample $\left(10 \times 2.5 \times 0.7 \mathrm{~mm}^{3}\right)$ with six In ohmic contacts, alloyed at $450^{\circ} \mathrm{C}$ for a few minutes in $\mathrm{N}_{2}$ flow, was used for the photocurrent $\left(I_{\mathrm{ph}}\right)$ and TSC measurements. The infrared light source was provided by a tungsten lamp ( $25 \mathrm{~W}$ ) filtered through a $\mathrm{Si}$ wafer, thus allowing photon energies less than $1.12 \mathrm{eV}$. The intensity of the light source could be varied about a factor of 3 . Both $I_{\mathrm{ph}}$ at $90 \mathrm{~K}$ and the TSC in a thermal scan were measured under $a$ bias of $20 \mathrm{~V}$ by an electrometer (Keithley 616), then stored and processed by a personal computer via an $\mathrm{A} / \mathrm{D}$ convertor (Keithley 197). To keep the same initial conditions for both $I_{\mathrm{ph}}$ and TSC measurements, the sample was always quickly cooled down from 310 to $90 \mathrm{~K}$ in the dark. The photoquenching of $I_{\mathrm{ph}}$ at $90 \mathrm{~K}$ was recorded over an interval of $200 \mathrm{~s}$ under different light intensities. To measure the photoquenching of the TSC spectral features, the sample was exposed to infrared light various times from 1 to $360 \mathrm{~s}$ in different runs, then heated from 90 to $270 \mathrm{~K}$ at a rate of $0.2 \mathrm{~K} / \mathrm{s}$. To measure the thermal recovery of the TSC spectral features, the sample was first illuminated for $5 \mathrm{~min}$ at $90 \mathrm{~K}$ to ensure a complete quenching of EL2 and the quenchable TSC traps, then it was warmed up to a certain recovery temperature $T_{r}$, ranging from 90 to $150 \mathrm{~K}$. Next it was quickly cooled back to $90 \mathrm{~K}$, and finally it was exposed to the same infrared light for $1 s$ to fill the traps before the TSC thermal scan. To check the type ( $n$ or $p$ ) of the sample after the photoquenching of EL2, and the type of carriers released during the TSC scan, Hall effect and conductivity measurements were carried out by placing the sample on a small, rare-earth permanent magnet of 3/4-in.-diam with a magnetic field strength of $2.4 \mathrm{kG}$.

The whole photocurrent quenching process at $90 \mathrm{~K}$, shown in Fig. 1, consists of three portions: (1) a buildup of $I_{\mathrm{ph}}$ at the very beginning $(t<2 \mathrm{~s}$ ) to a magnitude proportional to the EL 2 concentration, (2) a quenching of $I_{\mathrm{ph}}$ by about two and a half orders of magnitude, first quickly and then more slowly, and (3) an enhancement of $I_{\mathrm{ph}}$ after a transition time $t_{T}$. The transition time, defined by the minimum in the $I_{\mathrm{bh}}$ versus time curve, was found to be 


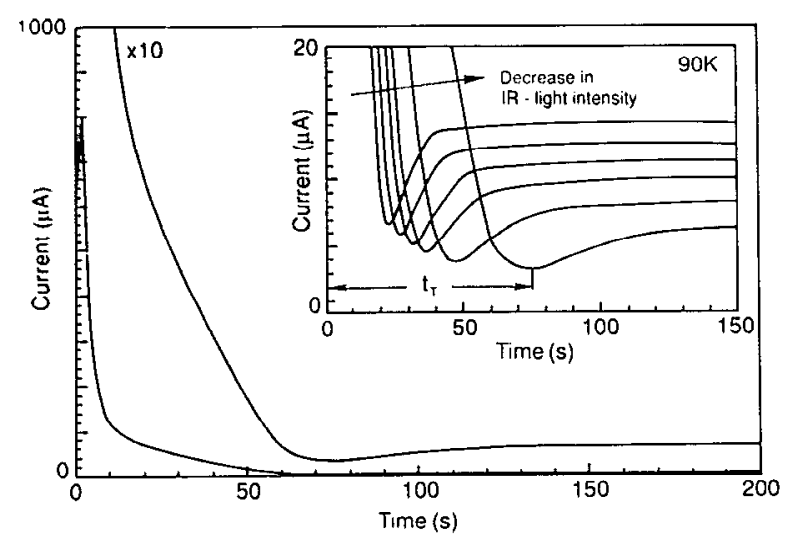

FIG. 1. Infrared quenching of the photocurrent at $90 \mathrm{~K}(h v \leqslant 1.12 \mathrm{eV})$, showing a minimum at a transition time $t_{T}$ which depends on the light intensity, as shown in the inset.

dependent on light intensity, as shown in the insert of Fig. 1. Furthermore, the product of $t_{T}$ and the corresponding $I_{\mathrm{ph}}$ at $300 \mathrm{~K}$ was found to be constant for different intensities. Since the $I_{\mathrm{ph}}$ at $300 \mathrm{~K}$ was shown to be proportional to the light intensity, the constancy of the product implies that the minimum in the curve is mainly sensitive to the total dose of absorbed photons. After the complete quenching of EL2, i.e., $t>t_{T}$, a photo-Hall measurement at $90 \mathrm{~K}$ indicated that the sample had converted from $n$ to $p$ type, with a hole concentration of $1.9 \times 10^{10} \mathrm{~cm}^{-3}$ and a hole mobility of $6500 \mathrm{~cm}^{2} / \mathrm{V} \mathrm{s}$.

The TSC spectra, obtained under a maximum light intensity and different illumination times, from 1 to $360 \mathrm{~s}$, are shown in Fig. 2. The spectral structure of the $1 \mathrm{~s}$ TSC spectrum (curve a in Fig. 2), referred to as TSC feature I, shows six peaks labeled $T_{2}, T_{3}, T_{4}, T_{5}, T_{5}^{*}$, and $T_{6}^{*}$; this structure is very similar to the TSC spectrum obtained by using $1.46 \mathrm{eV}$ light on the same sample and believed to be due to point defects. ${ }^{9}$ Their trap depths have been estimated to be $0.44 \mathrm{eV}\left(T_{2}\right), 0.39 \mathrm{eV}\left(T_{3}\right), 0.29 \mathrm{eV}\left(T_{4}\right), 0.27$ $\mathrm{eV}\left(T_{5}\right), 0.23 \mathrm{eV}\left(T_{5}^{*}\right)$, and $0.17 \mathrm{eV}\left(T_{6}^{*}\right)$ according to the

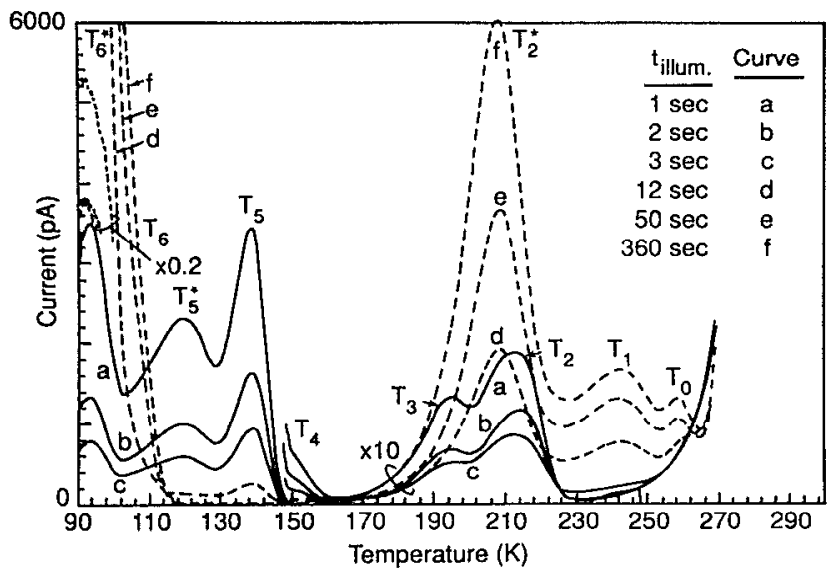

FIG. 2. TSC spectra under different illumination times from 1 to $360 \mathrm{~s}$, showing a drastic change in the TSC spectrum from feature I to II at $t_{\text {illum }} \sim 12 \mathrm{~s}$ (maximum light intensity used).

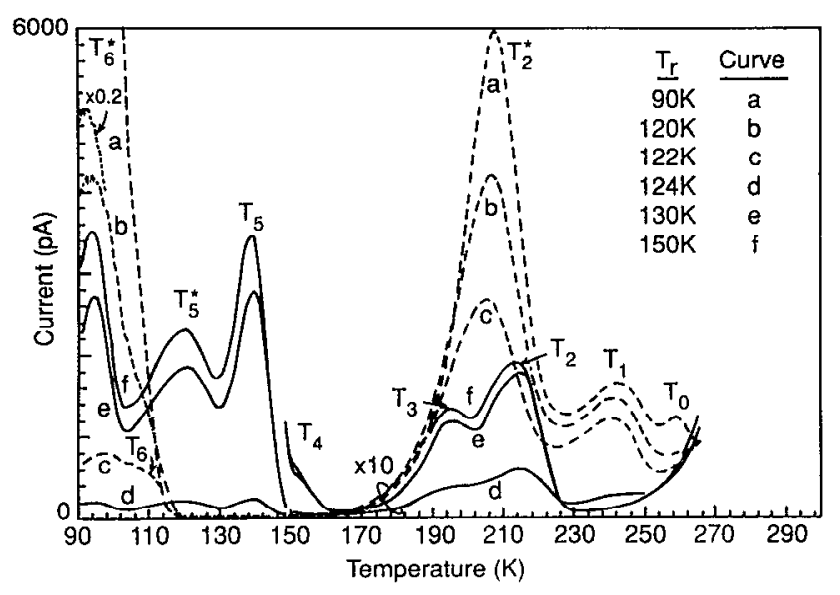

FIG. 3. TSC spectra following a soak at the different recovery temperatures $T_{r}$ 's and then a $1 \mathrm{~s}$ excitation at $90 \mathrm{~K}$ with the same light intensity as used in Fig. 2, showing a reversible change in TSC feature from II back to $I$ at $T,-124 \mathrm{~K}$.

peak positions and the heating rate. ${ }^{10}$ More accurate determinations of the trap depth for the deeper traps produce 0.49 and $0.42 \mathrm{eV}$ for $T_{2}$ and $T_{3}$, respectively. "Increasing the illumination time from 1 to $3 \mathrm{~s}$ did not change the basic structure of TSC, but caused a decrease in the peak height of all peaks. When the illumination time was increased to about $12 \mathrm{~s}$, a drastic change in the TSC spectral structure took place, i.e., a strong increase of $T_{6}^{*}$ with its peak position shifted to a lower temperature and the simultaneous transformation from $T_{2}$ and $T_{3}$ into $T_{0}, T_{1}$, and $T_{2}^{*}$; this new spectrum is referred to as TSC feature II. Further increase of the illumination time from 12 to $360 \mathrm{~s}$ did not alter the basic TSC spectral structure, but resulted in the enhancement of all TSC peaks to their saturation values. A new peak $T_{6}$, on the shoulder of the falling edge of $T_{6}^{*}$, can be observed as well. The trap depths in TSC feature II were determined to be $0.72 \mathrm{eV}\left(T_{0}\right), 0.61 \mathrm{eV}\left(T_{1}\right)$, and $0.44 \mathrm{eV}$ $\left(T_{2}\right)$. According to Hall effect measurements at the temperatures of the TSC peaks, it is likely that $T_{6}^{*}$ is an electron trap and $T_{2}^{*}, T_{1}$, and $T_{0}$ are hole traps; however, these assignments are not absolute.

TSC spectra following a soak at the recovery temperature $T_{n}$ and then a $1 \mathrm{~s}$ excitation with the same maximum light intensity at $90 \mathrm{~K}$, are shown in Fig. 3. When the selected $T_{r}$ 's were below $122 \mathrm{~K}$, the obtained TSC spectra were always in TSC feature II, with decreasing peak heights for increasing $T_{r}$ The existence of $T_{6}$ can be clearly seen in curves $\mathrm{b}\left(T_{r}=120 \mathrm{~K}\right)$ and $\mathrm{c}\left(T_{r}=122 \mathrm{~K}\right)$. However, when $T_{r}$ was increased to $124 \mathrm{~K}$, a dramatic and reversible change in the TSC spectral structure took place, i.e., the transformation of TSC feature II back into TSC feature I. Note that the temperature of this transformation is in good agreement with the temperature at which EL2* starts to recover back to EL2. ${ }^{1,4}$ Further increase of $T_{r}$ to $150 \mathrm{~K}$ did not change the TSC spectral structure but led eventually to a spectrum almost identical to that observed during EL2 quenching process after an identical $1 \mathrm{~s} \mathrm{exci-}$ tation (compare curve $f$ in Fig. 3 with curve a in Fig. 2).

The use of infrared light with lower intensity has no 


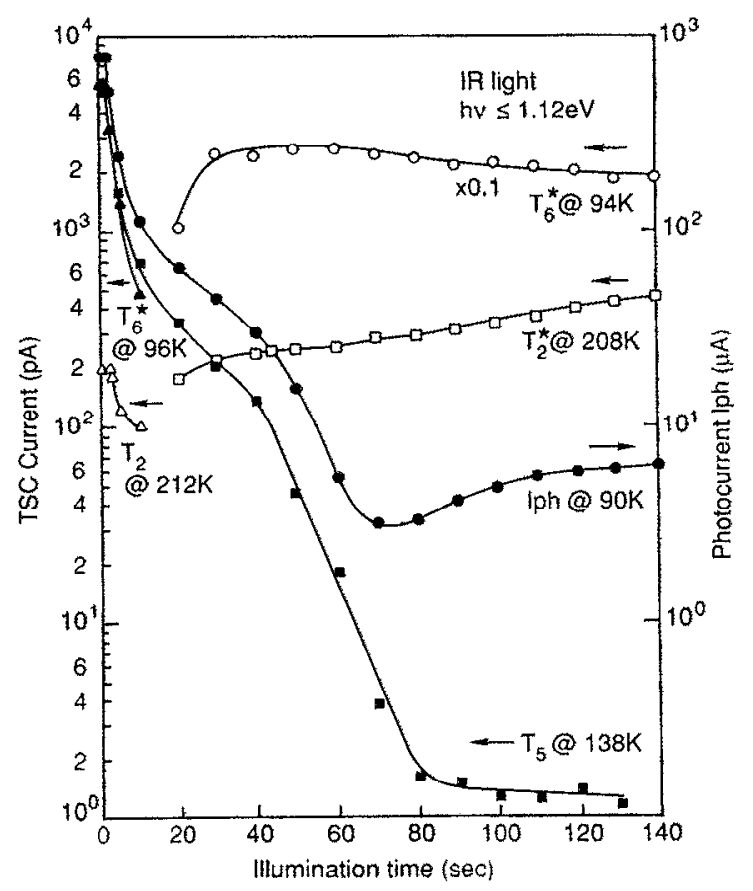

FIG. 4. Relationship between the quenched photocurrent and the quenched and enhanced TSC peaks for the main traps in TSC features I and II (minimum light intensity used).

effect on the observation of the two TSC features and their transformation, but retards the progress of the quenching and makes it easier to follow. A detailed relationship between the quenched $I_{\mathrm{ph}}$ and the quenched and enhanced TSC peaks for the main traps in TSC features I and II was measured by using a minimum light intensity $\left(t_{T}=75 \mathrm{~s}\right)$ and is plotted in Fig. 4. From the figure, it can be seen that (1) when $t<10 \mathrm{~s}$, the decrease in the peak height for the main TSC peaks $T_{5}$ and $T_{6}^{*}$ follows the quenched $I_{\mathrm{ph}}$ very well; (2) when $t_{T}>t>10 \mathrm{~s}$, the decay of $T_{5}$ still follows the quenched $I_{\mathrm{ph}}$ in spite of the transformation of the TSC feature from I to II at some time between 10 and $20 \mathrm{~s}$; and (3) when $t>t_{T}$, the enhancements of $T_{2}^{*}$ and $I_{\mathrm{ph}}$ seem to match with each other fairly well.

The TSC peak height for a given trap will depend on carrier (electron or hole) excitation rate, carrier lifetime, trap density, thermal emission rate, and sample heating rate. ${ }^{12}$ The latter two are constant but the others must all be considered. The similarities between the initial illumination-time dependences of the various TSC signals and $I_{\text {ph }}$, as seen in Fig. 4, leave no doubt that EL2 is a prime factor in the quenching process, since $I_{\mathrm{ph}}$ is mainly governed by EL2 for $t_{\text {illum }}<60 \mathrm{~s}$. Specifically, while EL2 is largely in its ground state, electron excitation will be dorminant, since the cross section for excitation of electrons by $1.12 \mathrm{eV}$ light is much larger than that for holes. Thus, electron traps should be favored for short illumination times. However, as EL2 converts to EL2*, other traps become more important in the carrier excitation process, and in fact, it is known from Hall-effect measurements that holes begin to dominate $I_{\mathrm{ph}}$ for long illumination times. From these considerations alone we would expect the traps that diminish with illumination time, $T_{5}^{*}, T_{5}, T_{4}, T_{3}$, and $T_{2}$ to be electron traps, and those that ircrease, $T_{2}^{*}, T_{1}$, and $T_{0}$, to be hole traps.

If this model is correct, then we should be able to fill and observe the quenched traps $T_{5}, T_{5}, T_{4}, T_{3}$, and $T_{2}$ by providing a source of electrons. This experiment is easily accomplished by removing the $\mathrm{Si}$ filter from the light source and thus applying above-band gap, white light after the sample has been quenched with the IR light. However, even after $1-300 \mathrm{~s}$ of the white light (about $10 \mathrm{~W}$ ), none of the quenched traps could be seen and the spectrum remained feature II. The same experiment was tried with $1 \mathrm{~s}$ illumination from a $1.46 \mathrm{eV}$ laser diode $(100 \mathrm{~mW})$ after IR quenching, and again none of the quenched traps reappeared. After $600 \mathrm{~s}$ of the $1.46 \mathrm{eV}$ light, however, the spectrum had returned to the unquenched feature 1 , but by then $I_{\mathrm{ph}}$ had recovered also, suggesting that EL2* had been optically transformed back to EL2. Thus, we believe the evidence indicates that traps $T_{5}^{*}, T_{5}, T_{4}, T_{3}$, and $T_{2}$ themselves undergo a transformation, as EL2 is transformed to EL2*, that causes them to disappear from the TSC spectrum. It is possible that some of them reappear as $T_{2}, T_{1}$, and/or $T_{0}$ in the quenched spectrum, e.g., it may be significant that $T_{2}^{*}$ is very close in energy to $T_{3}$ and $T_{2}$. We believe that these traps constitute point defects or impurities that are complexed with EL2 or $\mathrm{As}_{\mathrm{G}^{\mathrm{x} x}}$. Only in this case could they be so closely aligned with both the EL2 quenching and recovery. However, their exact microscopic natures must await further study.

After this manuscript was nearly complete, similar results were reported in the literature. ${ }^{13}$ Some of the main conclusions are the same although they did not see the strong peak $T_{2}$ arise at $208 \mathrm{~K}$ in the quenched spectrum. We believe the difference may be that our semi-insulating sample was Ga rich while theirs was probably As rich, as is standard.

ZQF was supported by ONR contract N0014-90-J11847 and DCL by USAF contract F33615-86-C01062. We would like to thank M. Mier for the EL2 absorption measurements.

'G. Vincent, D. Bois and A. Chantre J. Appl. Phys. 53, 3643 (1982).

${ }^{2}$ P. Layral, G. Vincent, A. Nouailhat, and G. Guillot, Solid State Commun. 42, 67 (1982).

${ }^{3}$ S. Nojima, J. Appl. Phys. 58. 3485 (1985).

${ }^{4}$ D. W. Fischer, Phys. Rev. B 37, 2968 (1988).

${ }^{5}$ II. J. Von Bardeleben, N. T. Bagraev, and J. C. Bourgoin, Appl. Phys. Lett. 51, 1451 (1987)

${ }^{6}$ M. O. Manasreh and D.W. Fischer, Phys. Rev. B 40, 11756 (1989).

${ }^{7}$ M. Baeumler, U. Kaufman, P. Mooney, and J Wagner, in Semi Insulating III-V Materials Toronto, 1990, edited by A. G. Milnes and C. Miner (Adam Hilger, Bristol, 1990), p. 29.

${ }^{8}$ M. Hoinkis and E. R. Weber, in Semi-Insulating III-V Materials, Malmö, 1988, edited by G. Grossman and L. Ledebo (Hilger, Bristol, 1988), p. 43 .

${ }^{7}$ Z-Q. Fang and D. C. Look, J. Appl. Phys. 69. June 15 (1991).

${ }^{i 0} \mathrm{Z}$,Q. Fang. L. Shan, 'T. E. Schlesinger, and A. G. Milnes, Mater, Sci. Eng. B 5, 397 (1990)

${ }^{1}$ Z-Q. Fang, L. Shan, T. E. Schlesinger, and A. G. Milnes, Mater. Res. Soc. Symp. Proc. 163, 189 (1990).

${ }^{12}$ D. C. Look, Semiconductors and Semimetals 19, 75 (1983).

${ }^{13}$ U. V. Desnica, D. I. Desnica, and B. Santic, A.ppl. Phys. Lett. 58, 278 (1991). 